\title{
An automated method for the evaluation of the pointing accuracy of Sun-tracking devices
}

\author{
Dietmar J. Baumgartner ${ }^{1}$, Werner Pötzi ${ }^{1}$, Heinrich Freislich ${ }^{1}$, Heinz Strutzmann ${ }^{1}$, Astrid M. Veronig ${ }^{1,2}$, and \\ Harald E. Rieder R $^{3,2,4}$ \\ ${ }^{1}$ Kanzelhöhe Observatory for Solar and Environmental Research (KSO), University of Graz, Treffen, Austria \\ ${ }^{2}$ Institute for Geophysics, Astrophysics and Meteorology/Institute of Physics (IGAM/IP), University of Graz, Graz, Austria \\ ${ }^{3}$ Wegener Center for Climate and Global Change (WEGC), University of Graz, Graz, Austria \\ ${ }^{4}$ Austrian Polar Research Institute (APRI), Vienna, Austria \\ Correspondence to: Dietmar J. Baumgartner (dietmar.baumgartner@uni-graz.at)
}

Received: 26 April 2016 - Discussion started: 6 September 2016

Revised: 20 February 2017 - Accepted: 23 February 2017 - Published: 22 March 2017

\begin{abstract}
The accuracy of solar radiation measurements, for direct (DIR) and diffuse (DIF) radiation, depends significantly on the precision of the operational Sun-tracking device. Thus, rigid targets for instrument performance and operation have been specified for international monitoring networks, e.g., the Baseline Surface Radiation Network (BSRN) operating under the auspices of the World Climate Research Program (WCRP). Sun-tracking devices that fulfill these accuracy requirements are available from various instrument manufacturers; however, none of the commercially available systems comprise an automatic accuracy control system allowing platform operators to independently validate the pointing accuracy of Sun-tracking sensors during operation. Here we present KSO-STREAMS (KSO-SunTRackEr Accuracy Monitoring System), a fully automated, systemindependent, and cost-effective system for evaluating the pointing accuracy of Sun-tracking devices. We detail the monitoring system setup, its design and specifications, and the results from its application to the Sun-tracking system operated at the Kanzelhöhe Observatory (KSO) Austrian radiation monitoring network (ARAD) site. The results from an evaluation campaign from March to June 2015 show that the tracking accuracy of the device operated at KSO lies within BSRN specifications (i.e., 0.1 ${ }^{\circ}$ tracking accuracy) for the vast majority of observations (99.8\%). The evaluation of manufacturer-specified active-tracking accuracies $\left(0.02^{\circ}\right)$, during periods with direct solar radiation exceeding $300 \mathrm{~W} \mathrm{~m}^{-2}$, shows that these are satisfied in $72.9 \%$ of observations. Tracking accuracies are highest during clear-
\end{abstract}

sky conditions and on days where prevailing clear-sky conditions are interrupted by frontal movement; in these cases, we obtain the complete fulfillment of BSRN requirements and $76.4 \%$ of observations within manufacturer-specified active-tracking accuracies. Limitations to tracking surveillance arise during overcast conditions and periods of partial solar-limb coverage by clouds. On days with variable cloud cover, $78.1 \%(99.9 \%)$ of observations meet active-tracking (BSRN) accuracy requirements while for days with prevailing overcast conditions these numbers reduce to $64.3 \%$ $(99.5 \%)$.

\section{Introduction}

A precise knowledge of the surface energy budget, which comprises the solar and terrestrial radiation fluxes, is essential for understanding Earth's climate system (e.g., Wild et al., 2015). The surface radiation budget itself is defined by the difference of the downward and upward components of short- and long wave irradiance (e.g., Augustine and Dutton, 2013). To date, ground-based measurements provide the most reliable information on short- and long wave irradiance. They are routinely utilized for retrieval optimization, the evaluation of satellite radiation products (Pinker et al., 2005; Gupta et al., 2004; Wang et al., 2014; Yan et al., 2011), and the evaluation and parameterization of radiative fluxes in global and regional climate models (e.g., Wild et al., 1998; 
Marty et al., 2003; Donner et al., 2011; Freidenreich and Ramaswamy, 2011) and reanalysis products (e.g., Allan, 2000).

Driven by the increasing need for high-accuracy surface radiation data for scientific and technical applications, e.g., to enhance the performance of solar photovoltaic plants (e.g., Fontani et al., 2011), national and international radiation monitoring networks have been established over recent decades. The most prominent international radiation monitoring network is the so-called Baseline Surface Radiation Network (BSRN) operating under the auspices of the World Climate Research Program (WCRP), e.g., Ohmura et al., (1998). BSRN sites are equipped with instruments of the highest accuracy. Targets for instrument performance and operation are specified in the BSRN guidelines (McArthur, 2005). Furthermore, BSRN guidelines are (closely) adopted by national radiation monitoring networks, e.g., ARAD (Austrian radiation monitoring network) in Austria (Olefs et al., 2016), SACRaM in Switzerland (Wacker et al., 2011), or SURFRAD in the US (Augustine et al., 2005).

BSRN guidelines require the operation of radiation sensors on Sun-tracking devices with specified accuracy, available, in various designs, from different instrument manufacturers. BSRN guidelines recommend the use of (i) single-axis synchronous-motor tracking devices, (ii) dual-axis passivetracking (algorithm-controlled) devices, or (iii) dual-axis active-tracking (quadrant-sensor-controlled) devices. For a detailed overview about advantages and disadvantages of these tracking devices, we refer the interested reader to Sect. 4 in McArthur (2005).

Among the suite of solar radiation measurements, the accuracy of pyrheliometer (direct solar radiation, DIR) and pyranometer measurements (diffuse solar radiation, DIF) depends significantly on the accuracy of the operational Suntracking device. Precise alignment is of the highest priority for the monitoring of DIF, as small misalignments can significantly affect the measurement accuracy. For measurements of DIF, one strives to solely shade the pyranometer's glass dome to mask as little of the diffuse component as possible while simultaneously shielding direct solar irradiance. As the BSRN network strives to achieve measurements at the highest possible accuracy, its guidelines recommend using a Sun-tracking device with an accuracy of $\pm 0.1^{\circ}$ or better (McArthur, 2005). For the monitoring of DIR, pointing accuracy is also important, but maybe less crucial than for DIF. This has been addressed in a study by Major, presented in Annex D of McArthur (2005), concluding that pointing errors are (i) negligible if smaller than a pyrheliometer's slope angle, but (ii) increasingly important with increasing error as measured irradiance decreases rapidly with increasing mispointing. Furthermore, BSRN guidelines recommend that tracking is monitored using a four-quadrant sensor, as the pointing accuracy is important in determining the quality of the direct beam measurement (McArthur, 2005).

Sun-tracking devices fulfilling these BSRN recommendations are available from various instrument manufacturers.
Nevertheless, none of the commercially available platforms comprise an automatic accuracy control system allowing platform operators to check whether the operational pointing accuracy of the Sun-tracking device indeed fulfills BSRN targets. The lack of a pointing accuracy control system is not unique to Sun-trackers used to accommodate solar radiation sensors. In fact, the determination of pointing accuracy is a common challenge for most types of Sun-pointing instruments, and various approaches have been presented to address this issue. For example, innovative camera-based (e.g., Gisi et al., 2011) and camera-free (e.g., Reichert et al., 2015) approaches to monitor tracking accuracy have been presented in the field of solar Fourier transform infrared (FTIR) spectrometry. While Gisi et al. (2011) documents a camera setup with real-time image-evaluation and tracking software (CamTrack), Reichert et al. (2015) presents an approach based on subsequent FTIR measurements with a different orientation of the solar rotation axis relative to the zenith direction, which allows us to obtain both mispointing components, the component in the zenith direction, and the component perpendicular to the solar rotation axis.

Here we present a related, camera-based, fully automated, system-independent, and cost-effective observing system to determine the pointing accuracy of Sun-tracking devices used in solar radiation monitoring networks which can be easily added to existing monitoring platforms, and its application to the Sun-tracking device operated at the Kanzelhöhe Observatory (KSO), Austria. We note that KSO-STREAMS (KSO-SunTRackEr Accuracy Monitoring System) is solely intended to monitor tracking accuracy and not to adjust the alignment of an operational Sun-tracking device.

\section{The proposed observing system for the evaluation of Sun-tracking device pointing accuracy}

\subsection{Components and installation}

The proposed observing system for continuous monitoring of the alignment (i.e., pointing accuracy) of the Sun-tracking device, hereinafter referred to as KSO-STREAMS, consists of five key components: (i) a circular aperture, (ii) an optical filter block, (iii) an achromatic lens (fixed focal length of $60 \mathrm{~mm}$ ), (iv) an adapted compact network camera with corresponding web connectivity, and (v) a fitted housing and mounting system. The observing system and a schematic illustration of the system components are shown in Fig. 1a and b. Details on system components are provided in Table 1 . During operation KSO-STREAMS needs to be mounted like a pyrheliometer on the Sun-tracking device (Fig. 1c) to ensure correct imaging of the Sun's position as identified by the tracker (computed and adjusted in the case of a four-quadrant sensor correction). The focal length of KSO-STREAMS is chosen to allow the registration of a misalignment of the imaged solar disk of up to $0.5^{\circ}$ (corresponding to approximately 
Table 1. Components of the KSO-STREAMS device and their characteristics.

\begin{tabular}{ll}
\hline Component & Characteristics \\
\hline $\begin{array}{l}\text { Circular aperture } \\
\text { Filter block }\end{array}$ & $\begin{array}{l}\text { Entrance window with a diameter of } 12 \mathrm{~mm} \\
\text { Combination of neutral- and gold-coated bandpass filter to } \\
\text { prohibit detector saturation }\end{array}$ \\
$\begin{array}{l}\text { Achromatic lens } \\
\text { Famera }\end{array}$ & $\begin{array}{l}\text { Compact length of } 60 \mathrm{~mm} \\
\text { resolution; } 1 / 4^{\prime \prime} \text { CMOS-sensor; adapted to Power over } \\
\text { Ethernet (PoE); special housing for outdoor usage }\end{array}$ \\
Housing and & $\begin{array}{l}\text { Meet requirements of IP65 and is ready to install } \\
\text { like a pyrheliometer on a Sun-tracking device }\end{array}$ \\
\hline
\end{tabular}

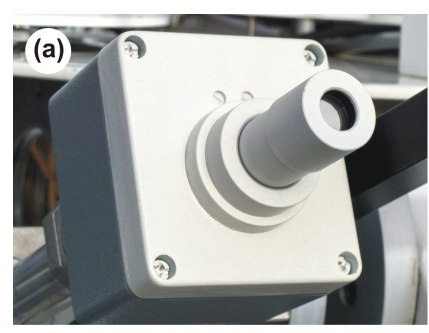

(b)
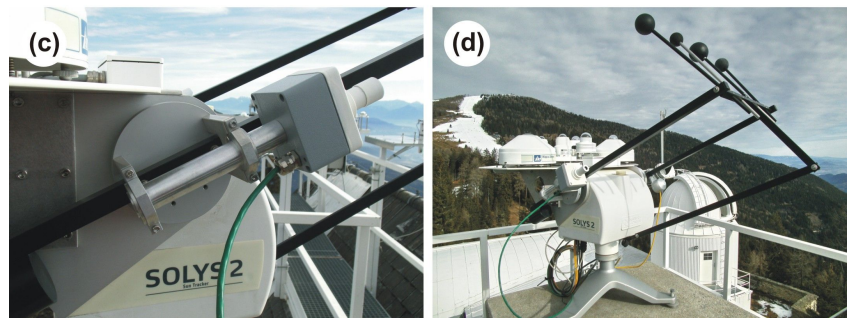

Figure 1. The instrumental setup is described as follows: (a) operating device; (b) optical layout with the following labeled components: circular aperture (A), filter block $(\mathrm{F})$, achromatic lens $(\mathrm{L})$, and camera chip (C); (c) mounting system; (d) radiation platform with KSO-STREAMS mounted on the Sun-tracking device (SOLYS2, Kipp \& Zonen) at the Kanzelhöhe Observatory ARAD site.

two solar radii) in each direction from the image center (see Fig. 2a). Fortunately, such severe misalignment hardly occurs in commercial Sun-tracking devices. Nevertheless, choosing such a wide range allows us to even quantify severe misalignments and enables us to identify the pointing accuracy of the Sun-tracking device and to quantify potential misalignments.

\subsection{Principle and defined accuracy limits}

KSO-STREAMS is operated by an automated script and takes, between sunrise and sunset, a snapshot every $15 \mathrm{~s}$ of the solar disk. The images taken are immediately processed as detailed below. A typical image taken by KSOSTREAMS is given in Fig. 2b. We note that high image contrast and minimal stray light, not image type (i.e., color), are important in further steps for solar-limb detection. Thus all KSO-STREAMS pictures are first converted to grayscale
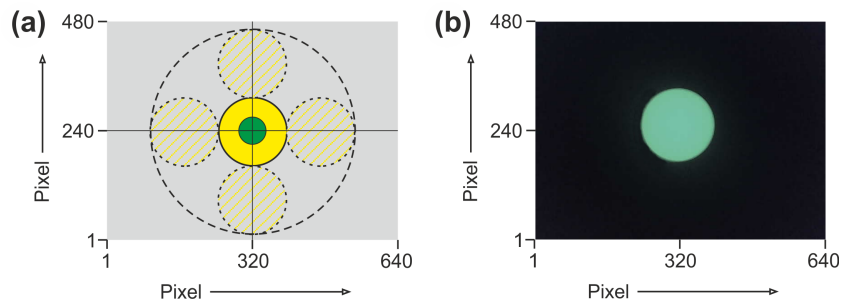

Figure 2. Solar disk images on the sensor array (VGA resolution) of the compact network camera of KSO-STREAMS. (a) Range of the detectable free movement of the positions of solar disk images within the detector array due to possible misalignment of the Suntracking device (the yellow area is the optimal position of the solar disk image, the green marked zone is the possible center of the solar disk image to be within BSRN requirements, and the yellow shaded area is the detectable misalignment of the Sun-tracking device through KSO-STREAMS); (b) typical solar disk image under clear-sky conditions.

(see Fig. 3a) and derotated during post-processing. Derotation is necessary to convert image pixel coordinates into azimuth and zenith coordinates, as it is not possible to mount KSO-STREAMS in perfect horizontal alignment on the Suntracking system. To determine the amount of image rotation necessary to achieve the horizontal alignment, we follow a four-step procedure: (i) the Sun-tracking device is positioned and fixed to its local noon position $3 \mathrm{~min}$ before actual local noon, (ii) images are recorded in $5 \mathrm{~s}$ intervals while the Sun is moving across the whole image plane, (iii) the center of the solar disk is determined (see method described below), and (iv) a line is fitted through the sequence of recorded solar disk centers, which represents the true solar path. Each picture has to be derotated for the angle between this fitted line and the image border to achieve horizontal image alignment.

For each image, the solar disk center ( $x-, y$-position) has to be determined prior to further processing. To this aim, we apply a standard Sobel operator (Jaehne, 1991) to the highcontrast images obtained in order to detect the solar limb. The Sobel operator calculates the image gradient of each pixel by convolving the image with a pair of $3 \times 3$ filters which 
(a)

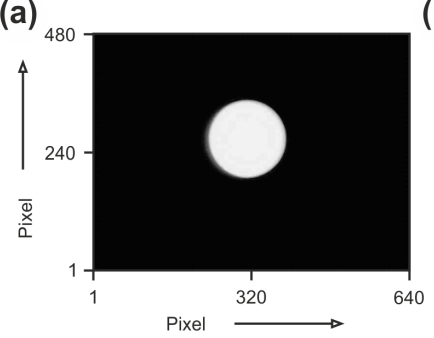

(b)

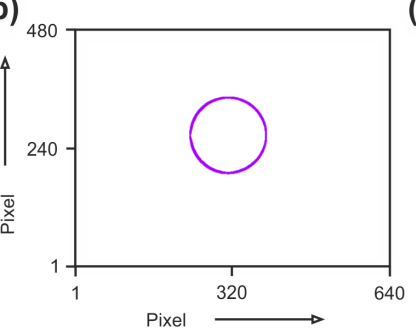

(c)

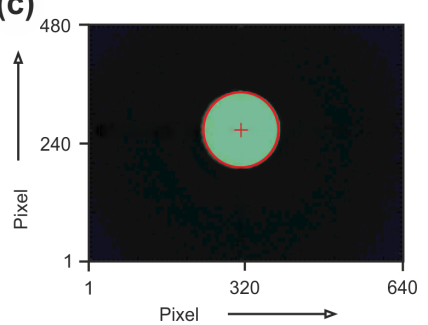

Figure 3. Illustration of individual steps in KSO-STREAMS image processing to derive a circular fit to solar-limb pixels: (a) grayscaletransformed solar disk image (original KSO-STREAMS picture is shown as background in panel c); (b) solar-limb pixels (purple) identified through the application of a Sobel operator to panel $\mathbf{a}$; and (c) the best fitting circle (red) to solar-limb pixels from panel $\mathbf{b}$ derived by a least-squares fitting approach superimposed on the original KSO-STREAMS picture. The red cross in panel $\mathbf{c}$ marks the center of the fitted circle.

estimate the gradients in the horizontal and vertical directions. The sum of the gradients in the horizontal and vertical directions yields the magnitude of the gradient. Given the high contrast between the Sun and background in KSOSTREAMS images, the solar limb is in first order defined through the pixels with the largest gradient (see Fig. 3b).

Next, we apply a classical least-squares circle fit (Ludwig, 1969) to the solar-limb pixels identified to calculate the radius and the center of the solar disk. Each of the first order solar-limb pixels identified is characterized through coordinates in the $x y$ plane, and the classical least-squares fitting approach minimizes the geometric distance of these points to the fitted circle.

The best fit through the set of $n$ points (i.e., the first order identified solar-limb pixels) is achieved through minimization of Eq. (1) by solving the system for $\frac{\partial F}{\partial h}=0, \frac{\partial F}{\partial k}=0$, and $\frac{\partial F}{\partial r}=0$ :

$F(h, k, r)=\sum_{i=1}^{n}\left[\sqrt{\left(x_{i}-h\right)^{2}-\left(y_{i}-k\right)^{2}}-r^{2}\right] \rightarrow \min$,

where $\left(x_{i}, y_{i}\right)$ denotes the first order solar-limb pixels, $(h, k)$ the circle center, and $r$ the radius of the fitting circle. As only coordinate pairs $\left(x_{i}, y_{i}\right)$ are known, the circle equation has to be linearized to obtain a series of linear equations yielding Eq. (4), which is linear in the undetermined coefficients $a, b$, and $c$, that allow us, once $a, b$, and $c$ are derived, to solve backwards for $h, k$, and $r$ :

$r^{2}=(x-h)^{2}+(y-k)^{2}=x^{2}-2 h x+h^{2}+y^{2}$ $-2 k y+k^{2}$

$x^{2}+y^{2}=2 h x+2 k y+r^{2}-h^{2}-k^{2}$,

$x^{2}+y^{2}=a x+b y+c$.

The coefficients $a, b$, and $c$ are derived by applying the matrix equation for a circular regression (Eq. 5):

$$
\left[\begin{array}{ccc}
\sum x_{i}^{2} & \sum x_{i} y_{i} & \sum x_{i} \\
\sum x_{i} y_{i} & \sum y_{i}^{2} & \sum y_{i} \\
\sum x_{i} & \sum y_{i} & n
\end{array}\right]\left[\begin{array}{c}
a \\
b \\
c
\end{array}\right]=\left[\begin{array}{c}
\sum x_{i}\left(x_{i}^{2}+y_{i}^{2}\right) \\
\sum y_{i}\left(x_{i}^{2}+y_{i}^{2}\right) \\
\sum x_{i}^{2}+y_{i}^{2}
\end{array}\right],
$$

where $n$ denotes the number of individual points $\left(x_{i}, y_{i}\right)$. It follows that a unique set of values for the coefficients $a, b$, and $c$ - that generate the circle of best fit - exist when the 3by-3 matrix on the left side of Eq. (5) is invertible. After deriving the coefficients $a, b$, and $c$, the center $(h, k)$ and radius $r$ can be computed through the following transformations:

$$
\begin{aligned}
& h=-\frac{a}{2}, \\
& k=-\frac{b}{2}, \\
& r=\frac{\sqrt{4 c+a^{2}+b^{2}}}{2} .
\end{aligned}
$$

Then, the best fitting circle can be established (see Fig. 3c).

The error on the circle fit has to be less than 1 pixel, considering the observing conditions (astronomical seeing). As the solar radius varies throughout the year uncertainty limits for the detection of the solar limb have been defined as $+5 \%$ of the largest and $-5 \%$ of the smallest astronomically calculated solar radius ( $\sim 76$ pixel and $\sim 74$ pixel, respectively) throughout the year. As we use a prime lens (i.e., a lens with a fixed focal length of $60 \mathrm{~mm}$ ), KSO-STREAMS focal length is not an issue. The results of the processing algorithm are stored in daily look-up tables for further post-processing and archived to allow retrospective investigation of the representativeness of solar radiation measurements found "dubious" in further analyses.

If both accuracy conditions are fulfilled, an image is considered valid and used for further analysis. It is obvious that turbidity and cloudiness (and here, especially broken cloud coverage in front of the Sun) complicate and compromise solar-limb detection. This is further investigated in Sect. 3.2, where we analyze Sun-tracker pointing accuracies over a wide range of cloud-cover conditions ranging from clear-sky to perpetual overcast. 

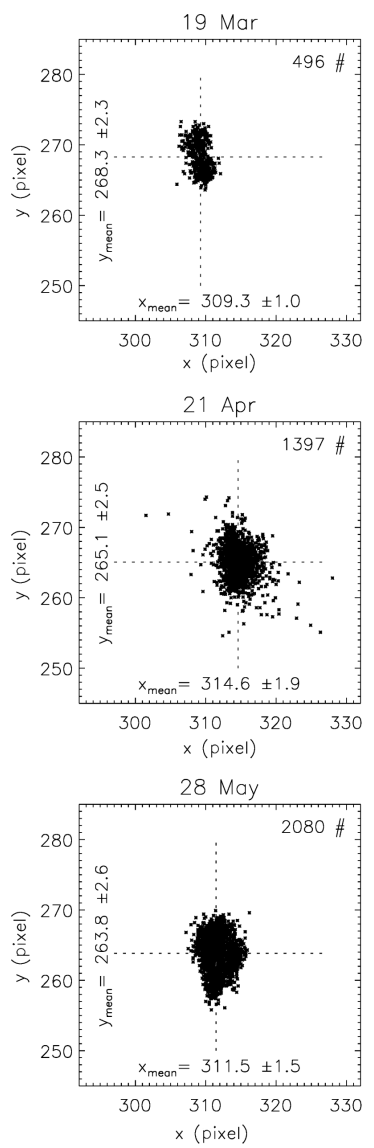
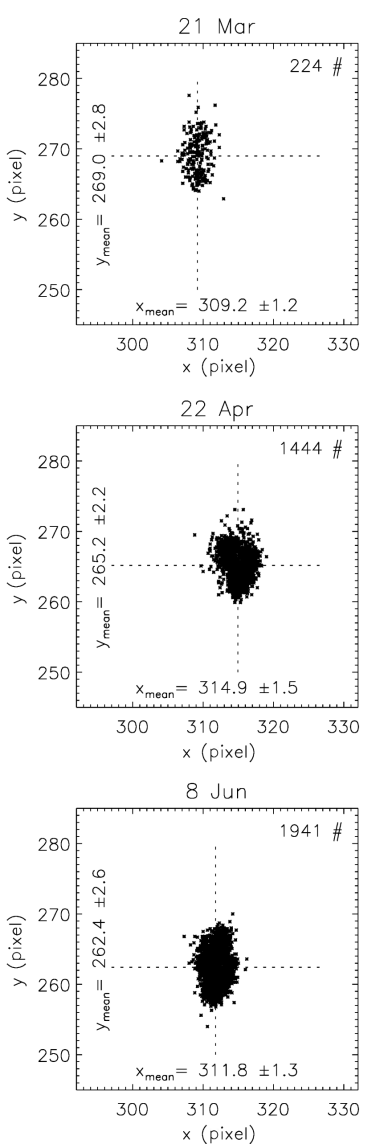
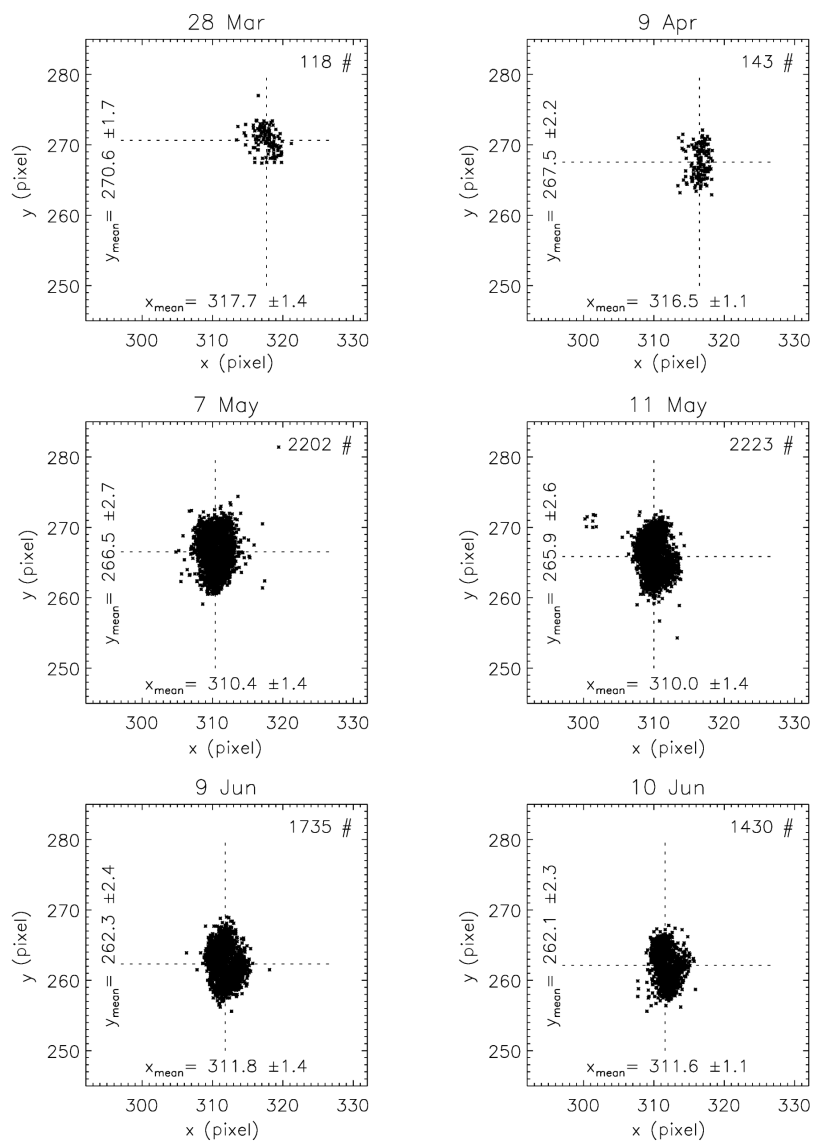

Figure 4. Solar disk center positions (black dots) determined by KSO-STREAMS, used for the determination of the mean daily zero-point centers on 12 clear-sky days in 2015. The zero-point center of KSO-STREAMS is defined as the mean value of these 12 zero-point centers.

\subsection{Initial zero-point determination of the solar center determined by KSO-STREAMS}

To determine the average solar disk center, we utilize data from 12 days from mid-March to mid-June 2015 (3 days each in March, April, May, and June; see Fig. 4) with perpetual clear-sky conditions and a high (and continuous) availability of observational data. Furthermore, we restrict the accuracy of limb detection to less than half a pixel. We then determine the average of the 12 individual daily-mean solar disk center positions and use this average as the initial zero point. We note that the difference among individual daily-mean zero positions (in both azimuthal and zenithal directions) is small, i.e., less than 8 pixels (which corresponds to approximately $0.03^{\circ}$ or $6 \%$ of the solar disk diameter), and is mainly affected by different atmospheric conditions (e.g., turbidity and humidity). In the following, the accuracy of the Sun-tracking device is characterized by the difference of KSO-STREAMS solar disk centers to this defined initial zero-point center.

\section{Application to the Sun-tracking device at the Kanzelhöhe Observatory, Austria}

\subsection{Field Measurements}

KSO-STREAMS was installed on 12 March 2015 on the Sun-tracking device (type SOLYS2, Kipp \& Zonen) of the Kanzelhöhe Observatory ARAD station (1540 ma.s.l.), see Fig. 1d. The Sun-tracking device is equipped with a Sun sensor which allows the fine-tuning of the alignment to the Sun if DIR is at least $300 \mathrm{~W} \mathrm{~m}^{-2}$. The information from the Sun sensor is updated every $10 \mathrm{~s}$ by the Sun-tracking device; thus, KSO-STREAMS is operated with $15 \mathrm{~s}$ "snapshots". Continuous operation (during ARAD site operation) started on 13 March 2015. Below, we detail the analysis of the Suntracking device performance and accuracy as monitored by KSO-STREAMS for 15 weeks during March to June 2015. 


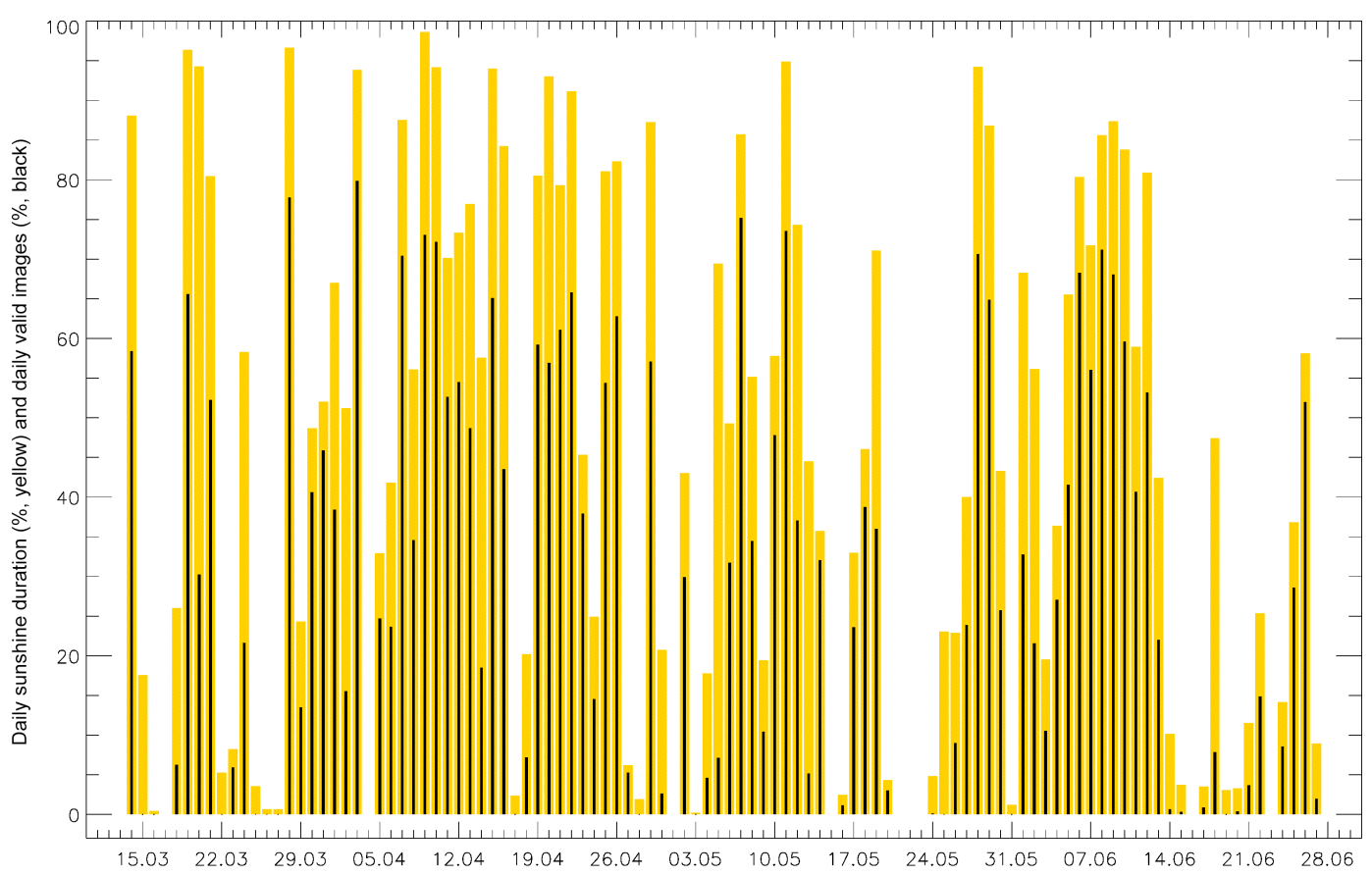

Figure 5. Relative daily sunshine duration (yellow) and valid KSO-STREAMS results (black) during the evaluation period from 14 March to 27 June 2015.

\subsection{Evaluation of the pointing accuracies of Sun-tracking devices under different meteorological conditions}

Over the 15-week evaluation period, a total of 100939 valid observations by KSO-STREAMS were available. This corresponds to $28 \%$ of the astronomically possible observations (360 228). The remaining observations ( $72 \%$ ) have been discarded due to exceedance of the accuracy requirements, detailed in Sect. 2.2. An overview of data availability (and relative sunshine duration) per day during the evaluation period is given in Fig. 5. We note that only $43.7 \%$ of the theoretically possible sunshine duration was observed during the evaluation period because of ambient weather conditions.

KSO-STREAMS allows identifying the fraction of observations within manufacturer-specified (i) active-tracking accuracy during periods with direct solar radiation exceeding $300 \mathrm{~W} \mathrm{~m}^{-2}$ and (ii) passive-tracking accuracy during periods with DIR below $300 \mathrm{~W} \mathrm{~m}^{-2}$. While the manufacturerspecified tracking accuracy deteriorates during periods with DIR below $300 \mathrm{~W} \mathrm{~m}^{-2}$ (passive tracking), this is not inherent to KSO-STREAMS observations. However, as the applied monitoring scheme is optical, it relies on the visibility of the solar disk; thus, no evaluation of the tracking accuracy is possible during periods of partial or full solar disk obstruction (see discussion below).

In the following, we illustrate the performance of the Suntracking device at the Kanzelhöhe Observatory in four selected situations, illustrating (i) nearly continuous clear sky, (ii) clear sky interrupted by frontal movement, (iii) variable cloud cover, and (iv) nearly perpetual overcast conditions.

Nearly continuous monitoring of the Sun-tracking device pointing accuracy was possible on 7 May 2015, with prevailing clear-sky conditions. Figure 6 shows the result of the zero-point distance determined by KSO-STREAMS (in $15 \mathrm{~s}$ intervals; panel a), and the result of direct solar radiation (panel b), derived from ARAD (1 min averages) and the actual total output of the Sun sensor of the Sun-tracking device (in $10 \mathrm{~min}$ increments) for this day. All available Sun disk centers, according to the selected restrictions (see Sect. 2.2), monitored on 7 May 2015 have been within the $0.1^{\circ}$ limit, as specified in the BSRN guidelines. A total of $90.7 \%$ of them have been within the manufacturer-specified active-tracking pointing accuracy of $0.02^{\circ}$. Individual Sun disk centers deviate from the overall set, triggered by individual clouds affecting the determination of the solar limb. Nevertheless, during these periods, the pointing accuracy of the Sun-tracking device lies well within manufacturer specifications for passivetracking $\left(0.1^{\circ}\right)$ and BSRN targets.

On 22 April 2015, zero-point center distances are comparable to 7 May 2015, although clear-sky conditions were interrupted through frontal movement from around 07:15 to 08:15 UT, indicated by the abrupt decline in DIR (Fig. 6d). No evaluation of the Sun-tracking device pointing accuracy was possible during the frontal passage as thick cloud coverage affected solar-limb detection. Before and after the frontal passage, clear skies prevailed and KSO-STREAMSmonitored pointing accuracies were within BSRN targets 

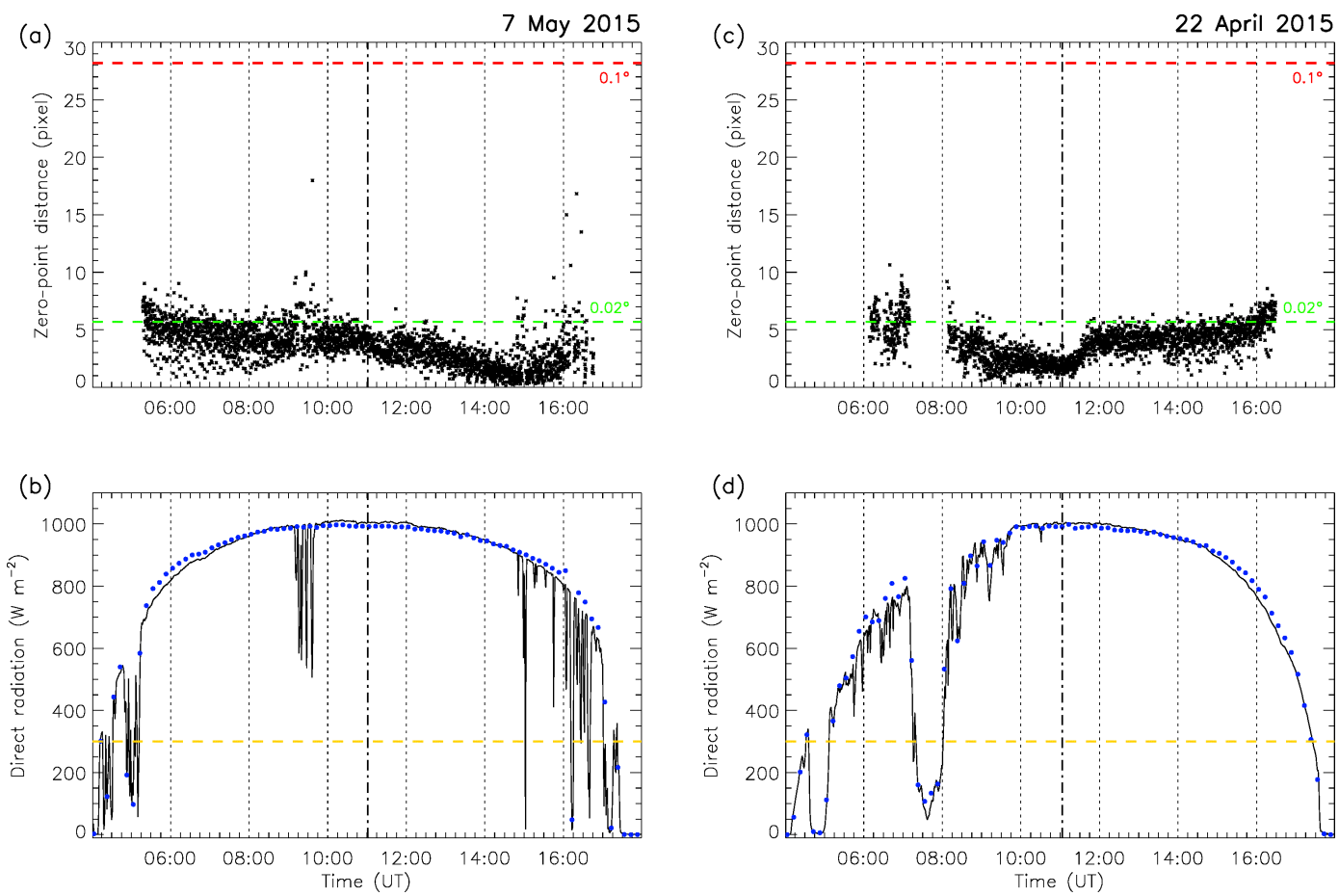

Figure 6. Performance of the Sun-tracking device at the Kanzelhöhe Observatory ARAD site for days with nearly continuous clear sky (7 May 2015, left column) and clear sky interrupted by frontal movement (22 April 2015, right column): (a) radial distance from the zero point of each valid image (asterisks); (b) direct radiation (1 min averages) derived from the Kanzelhöhe Observatory ARAD site (line) and from actual Sun-sensor measurements of the Sun-tracking device (in 10 min intervals, blue dots) on 7 May 2015. (c-d) As panels a-b but for 22 April 2015. Limits of active tracking (i.e., alignment within $0.02^{\circ}$ accuracy, green line) and passive tracking (i.e., alignment within $0.1^{\circ}$ accuracy, red line) are shown in panels a and $\mathbf{c}$. The yellow dashed line in panels $\mathbf{b}$ and $\mathbf{d}$ indicates the manufacturer-specified minimum of direct radiation $\left(300 \mathrm{~W} \mathrm{~m}^{-2}\right)$ needed for active-tracking mode. The vertical black dot-dashed line indicates the astronomical noon in all panels.

and largely (89.2\% of the observations) within manufacturer specifications for active tracking (see Fig. 6c).

Next, we focus on the evaluation of Sun-tracking accuracy during variable cloud cover as well as on days with nearly continuous cloud coverage, where active-tracking mode (manufacturer requirement is denoted by a total output of the Sun sensor of at least $300 \mathrm{~W} \mathrm{~m}^{-2}$ ) and, therefore, its evaluation is only possible during short temporal increments.

12 April and 10 May 2015 are representative for days with variable meteorological conditions, and, therefore, large variations in cloud cover. Periods with high (thick) cloud coverage, and limited direct radiation (Fig. 7b and d), affect KSOSTREAMS' ability of solar-limb detection. During times with thinner clouds, limb detection is possible, just as under the clear-sky conditions discussed above. Pointing accuracies are within manufacturer specifications for passive-tracking $\left(0.1^{\circ}\right)$ throughout, and consequently, also within BSRN targets. However, despite DIR exceeding manufacturer requirements for active tracking frequently on these days, only 68$78 \%$ of the valid observations are within specifications for active tracking (Fig. $7 \mathrm{a}$ and $\mathrm{c}$ ).
Similar results are found on days with prevailing overcast conditions, where only small gaps in cloud cover occur. Figure 8 shows data on pointing accuracy and direct radiation on 20 May and 3 June 2015, which are representative of overcast days during the evaluation period. On both days, the evaluation of the pointing accuracy of the Sun-tracking device was only possible during small gaps in cloud cover. We evaluate tracking accuracy within manufacturer targets for active tracking on these days, utilizing all observational data where the minimum of the ARAD direct radiation measurements (performed at a sample rate of $10 \mathrm{~Hz}$ ) within a minute exceeds $300 \mathrm{~W} \mathrm{~m}^{-2}$. On 20 May and 3 June $2015,42.6 \%$ and $49.2 \%$ of these selected observations, respectively, indeed fulfill the targeted accuracy of $\leq 0.02^{\circ}$.

If the analysis is extended to the entire 3-month period, we find that $96 \%$ of the observations (during periods where the minimum of the ARAD direct radiation measurements within a minute exceeds $300 \mathrm{~W} \mathrm{~m}^{-2}$ ) are within BSRN accuracy targets and about $75 \%$ are within manufacturerspecified active-tracking mode limits. While the vast majority of observations during active-tracking mode fulfills active-tracking accuracy requirements, difficulties arise dur- 

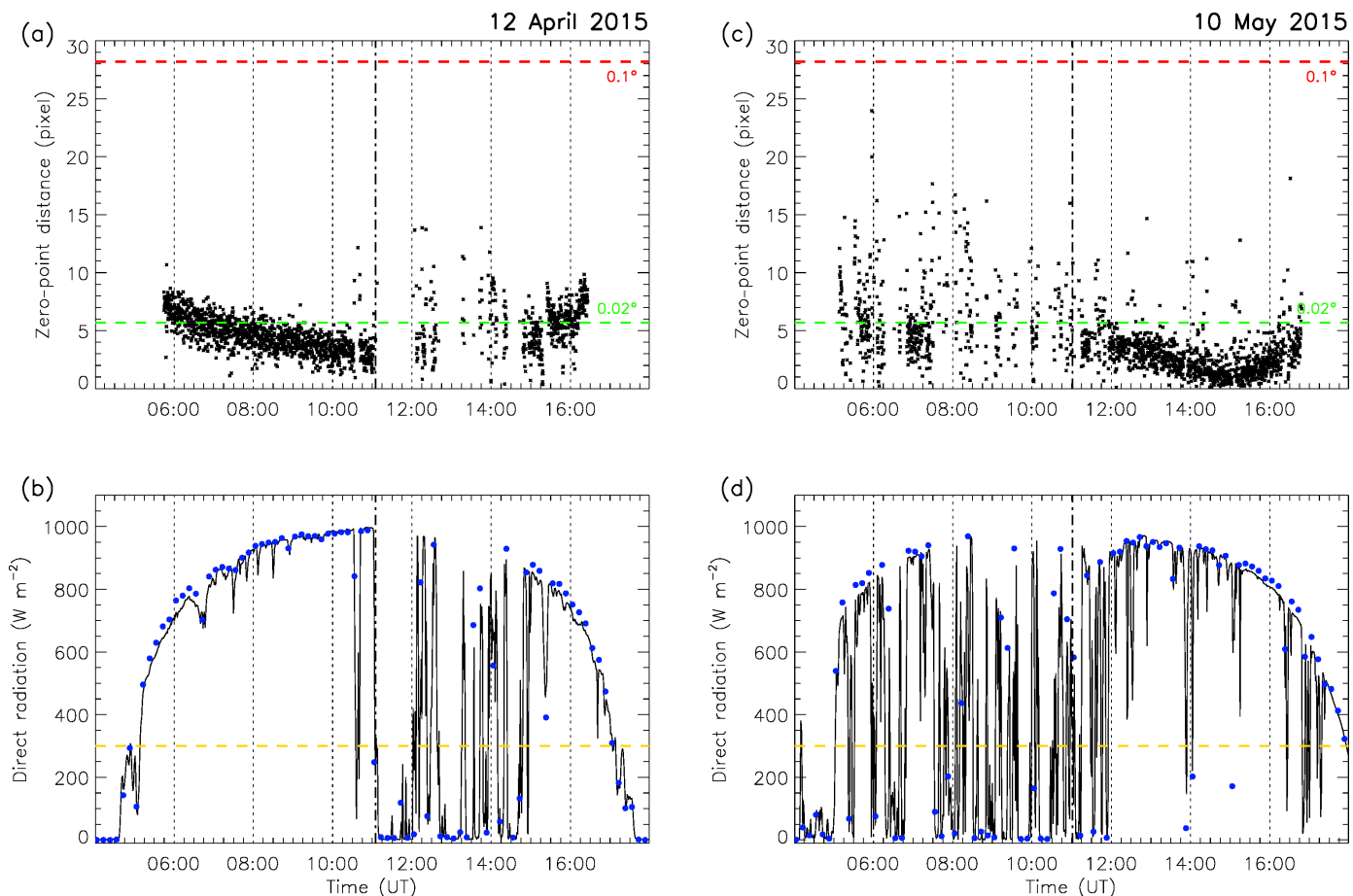

Figure 7. As Fig. 6, but for days with variable cloud cover (left, 12 April 2015; right, 10 May 2015).
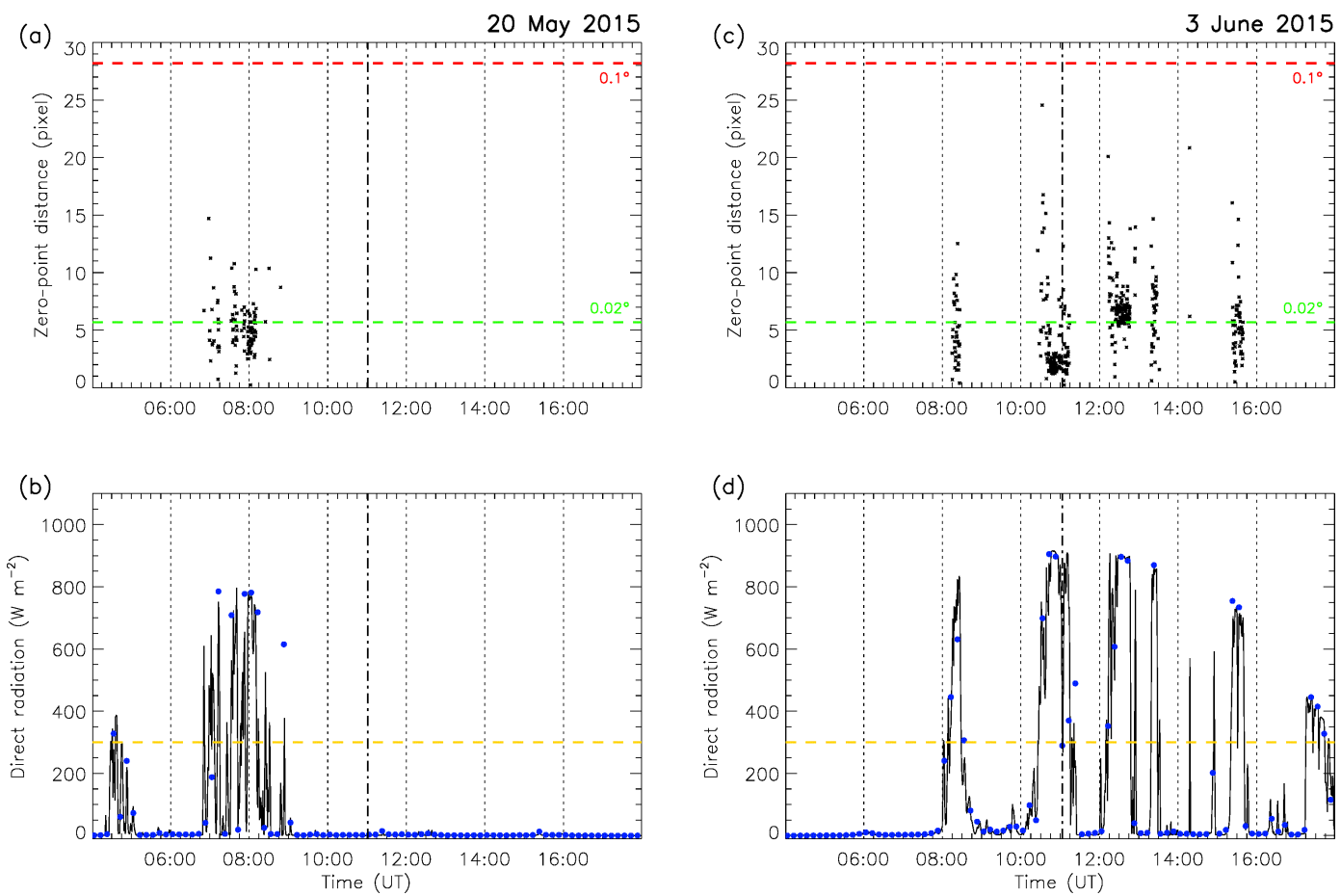

Figure 8. As Fig. 6, but for days with nearly perpetual overcast conditions (left, 20 May 2015; right, 3 June 2015).

ing breaks in overcast conditions. Nevertheless, it is important to note that even though larger fractions of the observations on days with overcast conditions do not fulfill activetracking requirements, they still fulfill BSRN targets.
Finally, we characterize the overall attainment of tracking accuracy within active-tracking targets on days comprising the sets of days with (i) nearly continuous clear sky, (ii) clear sky interrupted by frontal movement, (iii) variable 
Table 2. Summary of the achieved tracking accuracy for the determined sky-cover categories.

\begin{tabular}{|c|c|c|c|c|c|}
\hline Classification & $\begin{array}{l}\text { Relative amount of } \\
\text { valid observations }(V)\end{array}$ & $\begin{array}{l}\text { Active } \\
\text { tracking }\end{array}$ & $\begin{array}{r}\text { BSRN } \\
\text { requirement }\end{array}$ & $\begin{array}{r}\text { Number of valid } \\
\text { observations }\end{array}$ & $\begin{array}{r}\text { Number of } \\
\text { days }\end{array}$ \\
\hline $\begin{array}{l}\text { Nearly continuous clear sky or clear sky } \\
\text { interrupted by frontal movement }\end{array}$ & $V>65 \%$ & $76.4 \%$ & $100 \%$ & 33179 & 14 \\
\hline Variable cloud cover & $15 \%<V \leq 65 \%$ & $78.1 \%$ & $99.9 \%$ & 62735 & 46 \\
\hline Nearly perpetual overcast conditions & $V \leq 15 \%$ & $64.3 \%$ & $99.5 \%$ & 5025 & 25 \\
\hline Total & & $72.9 \%$ & $99.8 \%$ & 100939 & 85 \\
\hline
\end{tabular}

cloud cover, and (iv) nearly perpetual overcast conditions. On days with nearly continuous clear-sky conditions and with dominating clear-sky conditions interrupted by frontal movement, the BSRN targets are fully met, and the activetracking requirements are met for $76.4 \%$ of valid observations. Active-tracking accuracy requirements are less frequently met during variable cloud-cover conditions (defined as days with $\leq 65$ and $>15 \%$ of theoretically possible daily KSO-STREAMS observations). During the 3-month period, about half of the observational days (46 days) were categorized as "variable", and $78.1 \%(99.9 \%)$ of the observations fulfilled the active-tracking requirements (BSRN targets). Days with nearly perpetual overcast conditions are defined as days with $\leq 15 \%$ of the theoretically possible KSOSTREAMS observations. On these days, $64.3 \%$ of the available valid observations fulfilled active-tracking accuracy requirements, and $99.5 \%$ of the available valid observations fulfilled BSRN targets. Calculated over all 85 days with available measurements during the evaluation period, $72.9 \%$ of observations fulfill active-tracking accuracy requirements and $99.8 \%$ fulfill BSRN targets. A detailed summary of the achieved tracking accuracy (per category and total) is provided in Table 2.

\section{Conclusions}

Precise Sun-tracking is necessary for high-accuracy measurements of direct and diffuse solar radiation. Therefore, rigid targets for Sun-tracking pointing accuracies are specified in national and international radiation monitoring networks, e.g., the Baseline Surface Radiation Network, which specifies pointing accuracy requirements within $0.1^{\circ}$. Suntracking devices fulfilling this pointing accuracy are available from a variety of instrument manufacturers, but none of the commercially available instruments comprise an automatic monitoring system allowing station operators independent evaluation of pointing accuracies during operation. In this paper, we present KSO-STREAMS, a platform-independent, fully automated, and cost-effective system to evaluate the pointing accuracy of Sun-tracking devices. During operation, KSO-STREAMS is mounted like a pyrheliometer on the
Sun-tracking device to ensure correct imaging of the Sun's position, as identified by the tracking device

To determine the pointing accuracy of the Sun-tracking device operated at the Kanzelhöhe Observatory Austrian radiation monitoring network (Olefs et al., 2016) site, observations by KSO-STREAMS, taken over a 15 -week period from March to June 2015, were analyzed. Instrument performance was evaluated for valid KSO-STREAMS observations during four sets of ambient meteorological conditions: (i) nearly continuous clear sky, (ii) clear sky interrupted by frontal movement, (iii) variable cloud cover, and (iv) nearly perpetual overcast conditions. The results show that $72.9 \%$ of all observations made during periods with DIR more than $300 \mathrm{~W} \mathrm{~m}^{-2}$, fulfill manufacturer-specified active-tracking accuracy requirements $\left(0.02^{\circ}\right)$ and $99.8 \%$ fulfill BSRN targets $\left(0.1^{\circ}\right)$. On days with nearly continuous clear-sky conditions and/or clear-sky conditions interrupted by frontal movement, the BSRN requirements are fully satisfied and accuracies for active tracking are met for $76.4 \%$ of observations. Similar results are found for days with variable cloud-cover conditions. As expected, Sun-tracking pointing accuracies are lowest during days with nearly perpetual overcast conditions; here, $64.3 \%$ of observations meet active-tracking requirements. Nevertheless, the BSRN accuracy targets are still almost completely met $(99.5 \%)$, illustrating the strong performance of the Sun-tracking system operated at KSO (SOLYS2, Kipp $\&$ Zonen). The result of less accurate quadrant-sensor-based tracking on days with cloud influence is robust and not dependent on the KSO-STREAMS analysis algorithm, as all analyses were restricted to valid KSO-STREAMS observations. We conclude that KSO-STREAMS provides valuable information on the quality of radiation measurement accuracies through evaluation of the underlying pointing accuracies of the operational Sun-tracking device.

Data availability. The data presented in this article are available at www.kso.ac.at/publication_data/baumgartner_amt_2017/.

Competing interests. The authors declare that they have no conflict of interest. 
Acknowledgements. The authors thank three anonymous referees for their useful suggestions and their help in improving this article during the discussion phase.

Edited by: M. Van Roozendael

Reviewed by: three anonymous referees

\section{References}

Allan, R. P.: Evaluation of simulated clear-sky longwave radiation using ground-based observations, J. Climate, 13, 1951-1964, doi:10.1175/1520-0442(2000)013<1951:Eoscsl>2.0.Co;2, 2000.

Augustine, J. A. and Dutton, E. G.: Variability of the surface radiation budget over the United States from 1996 through 2011 from high-quality measurements, J. Geophys. Res.-Atmos., 118, 43-53, doi:10.1029/2012JD018551, 2013.

Augustine, J. A., Hodges, G. B., Cornwall, C. R., Michalsky, J. J., and Medina, C. I.: An update on SURFRAD - The GCOS Surface Radiation budget network for the continental United States, J. Atmos. Ocean. Tech., 22, 1460-1472, doi:10.1175/Jtech1806.1, 2005.

Donner, L. J., Wyman, B. L., Hemler, R. S., Horowitz, L. W., Ming, Y., Zhao, M., Golaz, J. C., Ginoux, P., Lin, S. J., Schwarzkopf, M. D., Austin, J., Alaka, G., Cooke, W. F., Delworth, T. L., Freidenreich, S. M., Gordon, C. T., Griffies, S. M., Held, I. M., Hurlin, W. J., Klein, S. A., Knutson, T. R., Langenhorst, A. R., Lee, H. C., Lin, Y. L., Magi, B. I., Malyshev, S. L., Milly, P. C. D., Naik, V., Nath, M. J., Pincus, R., Ploshay, J. J., Ramaswamy, V., Seman, C. J., Shevliakova, E., Sirutis, J. J., Stern, W. F., Stouffer, R. J., Wilson, R. J., Winton, M., Wittenberg, A. T., and Zeng, F. R.: The Dynamical Core, Physical Parameterizations, and Basic Simulation Characteristics of the Atmospheric Component AM3 of the GFDL Global Coupled Model CM3, J. Climate, 24, 34843519, doi:10.1175/2011jcli3955.1, 2011.

Fontani, D., Sansoni, P., Francini, F., Jafrancesco, D., Mercatelli, L., and Sani, E.: Pointing Sensors and Sun Tracking Techniques, Int. J. Photoenergy, 806518, doi:10.1155/2011/806518, 2011.

Freidenreich, S. M. and Ramaswamy, V.: Analysis of the biases in the downward shortwave surface flux in the GFDL CM2.1 general circulation model, J. Geophys. Res.-Atmos., 116, D08208, doi:10.1029/2010jd014930, 2011.

Gisi, M., Hase, F., Dohe, S., and Blumenstock, T.: Camtracker: a new camera controlled high precision solar tracker system for FTIR-spectrometers, Atmos. Meas. Tech., 4, 47-54, doi:10.5194/amt-4-47-2011, 2011.

Gupta, S. K., Kratz, D. P., Wilber, A. C., and Nguyen, L. C.: Validation of parameterized algorithms used to derive TRMM-CERES surface radiative fluxes, J. Atmos. Ocean. Tech., 21, 742752, doi:10.1175/1520-0426(2004)021<0742:Vopaut>2.0.Co;2, 2004.

Jaehne, B.: Digitale Bildverarbeitung, Springer, Berlin Heidelberg, 1991.
Ludwig, R.: Methoden der Fehler- und Ausgleichsrechnung, Vieweg, Braunschweig, 1969.

Marty, C., Philipona, R., Delamere, J., Dutton, E. G., Michalsky, J., Stamnes, K., Storvold, R., Stoffel, T., Clough, S. A., and Mlawer, E. J.: Downward longwave irradiance uncertainty under arctic atmospheres: Measurements and modeling, J. Geophys. Res.Atmos., 108, 4358, doi:10.1029/2002jd002937, 2003.

McArthur, L. J. B.: Baseline Surface Radiation Network (BSRN) Operations Manual Version 2.1WCRP-121, WMO/TD-No. 1274, Geneva, 2005.

Ohmura, A., Dutton, E. G., Forgan, B., Frohlich, C., Gilgen, H., Hegner, H., Heimo, A., Konig-Langlo, G., McArthur, B., Muller, G., Philipona, R., Pinker, R., Whitlock, C. H., Dehne, K., and Wild, M.: Baseline Surface Radiation Network (BSRN/WCRP): New precision radiometry for climate research, B. Am. Meteorol. Soc., 79, 2115-2136, doi:10.1175/15200477(1998)079<2115:Bsrnbw>2.0.Co;2, 1998.

Olefs, M., Baumgartner, D. J., Obleitner, F., Bichler, C., Foelsche, U., Pietsch, H., Rieder, H. E., Weihs, P., Geyer, F., Haiden, T., and Schöner, W.: The Austrian radiation monitoring network ARAD - best practice and added value, Atmos. Meas. Tech., 9, 15131531, doi:10.5194/amt-9-1513-2016, 2016.

Pinker, R. T., Zhang, B., and Dutton, E. G.: Do satellites detect trends in surface solar radiation?, Science, 308, 850-854, doi:10.1126/science.1103159, 2005.

Reichert, A., Hausmann, P., and Sussmann, R.: Pointing errors in solar absorption spectrometry - correction scheme and its validation, Atmos. Meas. Tech., 8, 3715-3728, doi:10.5194/amt-83715-2015, 2015.

Wacker, S., Grobner, J., Hocke, K., Kampfer, N., and Vuilleumier, L.: Trend analysis of surface cloud-free downwelling long-wave radiation from four Swiss sites, J. Geophys. Res.-Atmos., 116, D10104, doi:10.1029/2010jd015343, 2011.

Wang, P., Sneep, M., Veefkind, J. P., Stammes, P., and Levelt, P. F.: Evaluation of broadband surface solar irradiance derived from the Ozone Monitoring Instrument, Remote Sens. Environ., 149, 88-99, doi:10.1016/j.rse.2014.03.036, 2014.

Wild, M., Ohmura, A., Gilgen, H., Roeckner, E., Giorgetta, M., and Morcrette, J. J.: The disposition of radiative energy in the global climate system: GCM-calculated versus observational estimates, Clim. Dynam., 14, 853-869, doi:10.1007/s003820050260, 1998.

Wild, M., Folini, D., Hakuba, M. Z., Schar, C., Seneviratne, S. I., Kato, S., Rutan, D., Ammann, C., Wood, E. F., and KonigLanglo, G.: The energy balance over land and oceans: an assessment based on direct observations and CMIP5 climate models, Clim. Dynam., 44, 3393-3429, doi:10.1007/s00382-014-2430-z, 2015.

Yan, H. R., Huang, J. P., Minnis, P., Wang, T. H., and Bi, J. R.: Comparison of CERES surface radiation fluxes with surface observations over Loess Plateau, Remote Sens. Environ., 115, 14891500, doi:10.1016/j.rse.2011.02.008, 2011. 